\title{
Lebesgue Constant Minimizing Bivariate Barycentric Rational Interpolation
}

\author{
Qianjin Zhao* and Bingbing Wang* \\ School of Science, Anhui University of Science and Technology, Huainan 232001, P.R.China
}

Received: 5 Jun. 2013, Revised: 4 Oct. 2013, Accepted: 5 Oct. 2013

Published online: 1 Jan. 2014

\begin{abstract}
The barycentric form is the most stable formula for a rational interpolant on a finite interval. The choice of the barycentric weights can ensure the absence of poles on the real line, so how to choose the optimal weights becomes a key question for bivariate barycentric rational interpolation. A new optimization algorithm is proposed for the best interpolation weights based on the Lebesgue constant minimizing. Several numerical examples are given to show the effectiveness of the new method.
\end{abstract}

Keywords: Lebesgue constant, bivariate, barycentric rational interpolation, weight

\section{Introduction}

Interpolation is one of the important tools of mathematics. It can solve many original problems like differentiation, multistep methods for ordinary differential equations, collocation methods for partial differential equations, etc. So interpolation is a key subject in numerical analysis.

In recent years, barycentric rational interpolation has been one of the focuses for researchers [1,2]. As an important method for non-linear approximation, it possesses various advantages in comparison with other interpolation, such as small calculation quantity, good numerical stability, no poles and no unattainable points [3]. In 1945, W. Taylor discovered the barycentric formula for evaluating the interpolating polynomial [4]. In 1984, W. Werner presented the barycentric rational interpolation [5]. The barycentric rational interpolation can have no poles and no unattainable points by controlling the barycentric weights [6]. The barycentric rational interpolation is determined when its weights are given. How to choose the optimal weights becomes a key question for barycentric rational interpolation.

In the single-variable case this problem has been solved by using a quantity of different approaches. One of them, the minimizing Lebesgue constant approach, has been developed in a series of papers [6,7]. In 1996, Berrut obtained the optimal weights on a finite interval by minimizing the Lebesgue constant for the given nodes [6]. In this way, the linearity of the interpolation process with respect to the interpolated functions is preserved, and it can be written as function of barycentric weights. Lebesgue constant is based on Lagrange bases of polynomials. In this paper we study the problem of two-variable barycentric rational interpolation. The reason for our interest in this problem is that in a more general context, barycentric rational interpolation of multivariate functions play a central role in model reduction of linear systems which depend on parameters. A new optimization algorithm for bivariate barycentric rational interpolation is presented. A crucial optimization algorithm is obtained for the best interpolation weights based on the minimizing Lebesgue constant. Minimizing Lebesgue constant is been as the objective function, the weights are been as decision variables and satisfy some constraint conditions to ensure the bivariate barycentric rational function has no poles and no unattained points and uniqueness. The solution of the optimization model is obtained using the software LINGO.

The paper is structured as follows. Section 2 is dedicated to the barycentric rational interpolation. In Section 3 our main tool, the Lebesgue constant, is introduced by means of Lagrange bases of polynomials. In particular, after a brief overview of the single-variable case, the two-variable extension is developed. In Section 4, some numerical examples are presented which illustrate the effectiveness of the new method.

\footnotetext{
*Corresponding author e-mail: qianjinzhao@yahoo.com.cn; wangbingbing510811@163.com
} 


\section{Barycentric rational interpolation}

In 1984, Schneider and Werner have been the first to determine barycentric representations of rational interpolations.

\subsection{Univariate barycentric rational interpolation}

Given $n+1$ mutually distinct points $x_{0}, x_{1}, \cdots, x_{n}$ and function values $f_{0}, f_{1}, \cdots, f_{n}$, the rational functions

$$
r_{n}(x)=\frac{\sum_{i=0}^{n} \frac{w_{i}}{x-x_{i}} f_{i}}{\sum_{i=0}^{n} \frac{w_{i}}{x-x_{i}}}, \quad w_{i} \neq 0
$$

interpolate the values $f_{i}$ at the points $x_{i}$ for any nonzero weights $w_{i}$, in other words $r_{n}\left(x_{i}\right)=f_{i}$ [4].

From the above, as long as the weights are not all zero, it will not appear unattainable points, and it is easy to see that the degree in denominator and numerator of $r_{n}(x)$ is at most $n$ and the barycentric weights are only determined up to a multiplicative constant.

A necessary condition for the barycentric weights to satisfy $r_{n}(x)$ has no poles in $\left[x_{0}, x_{n}\right]$ is

$$
w_{i} \cdot w_{i+1}<0, \quad i=0,1, \cdots, n-1 .
$$

\subsection{Bivariate barycentric rational interpolation}

In this section, the barycentric rational interpolation is generalized to the two-variable case.

Lemma 2.1. [9]. Let $\prod_{x}^{m}=\left\{x_{i} \mid i=0,1, \cdots, m\right\} \subset(a, b)$,

$$
\begin{aligned}
& x_{0}<x_{1}<\cdots<x_{m}, \prod_{y}^{n}=\left\{y_{j} \mid j=0,1, \cdots, n\right\} \subset(c, d), \\
& y_{0}<y_{1}<\cdots<y_{n}, \text { and } \\
& \prod_{x, y}^{m, n}=\left\{\left(x_{i}, y_{j}\right) \mid i=0,1, \cdots, m, j=0,1, \cdots, n\right\} \\
& \quad \subset(a, b) \times(c, d), \\
& f\left(x_{i}, y_{j}\right)=f_{i, j}(i=0,1, \cdots, m, j=0,1, \cdots, n) .
\end{aligned}
$$

Then

$$
R(x, y)=\frac{\sum_{i=0}^{m} \frac{w_{i}}{x-x_{i}} r_{i}(y)}{\sum_{i=0}^{m} \frac{w_{i}}{x-x_{i}}}, \quad w_{i} \neq 0
$$

here

$$
r_{i}(y)=\frac{\sum_{j=0}^{n} \frac{u_{j}}{y-y_{j}} f_{i, j}}{\sum_{j=0}^{n} \frac{u_{j}}{y-y_{j}}}, \quad u_{j} \neq 0 .
$$

All the weights $w_{i}$ and $u_{j}$ are both nonzero so that the bivariate barycentric rational interpolant has no unattained points. And the weights $w_{i}$ and $u_{j}$ are chose for the rational function $r(x, y)$ has no poles in finite interval.

Lemma 2.2. [5]. Let $x_{0}<\cdots<x_{m}, w_{i} \neq 0, i=0, \cdots, n$, $y_{0}<\cdots<y_{n}, u_{j} \neq 0, j=0, \cdots, n$. If $r(x, y)$ has no poles in $D \subset\left(x_{0}, x_{m}\right) \times\left(y_{0}, y_{n}\right)$, then

$$
\begin{aligned}
& \operatorname{sign}\left(w_{i}\right) \cdot \operatorname{sign}\left(w_{i+1}\right)=-1, i=0,1, \cdots, m, \\
& \operatorname{sign}\left(u_{j}\right) \cdot \operatorname{sign}\left(u_{j+1}\right)=-1, j=0,1, \cdots, n .
\end{aligned}
$$

Furthermore, for ensuring the uniqueness of the optimization solution, we take the normative constraint

$$
\begin{aligned}
& \sum_{i=0}^{m}\left|w_{i}\right|=1 \\
& \sum_{j=0}^{n}\left|u_{j}\right|=1 .
\end{aligned}
$$

\section{Lebesgue constant minimizing bivariate barycentric rational interpolation}

Theorem 3.1. Let $x_{0}, x_{1}, \cdots, x_{n}$ be $n+1$ distinct points in $[a, b]$. The linear projection $P_{n}$ which in $C[a, b]$ associates with any function $f$ the polynomial $P_{n} f \in P_{n}$ interpolating $f$ between the $x_{k}$ 's has the norm

$$
\left\|P_{n}\right\|=\Lambda_{n}=\max _{a \leq x \leq b} \sum_{k=0}^{n}\left|l_{k}(x)\right|
$$

$\lambda_{n}(x)=\sum_{k=0}^{n}\left|l_{k}(x)\right|$ is called the Lebesgue function of the approximation operator and $\Lambda_{n}$ is Lebesgue constant [8].

From the above, $l_{k}(x)$ is Lagrange fundamental polynomial, it can be written as

$$
l_{k}(x)=w_{k}\left(l(x) /\left(x-x_{k}\right)\right)
$$

here $w_{k}=1 / \prod_{i=0, i \neq k}^{n}\left(x_{k}-x_{i}\right)$ and $l(x)=\left(x-x_{0}\right)\left(x-x_{1}\right) \cdots\left(x-x_{n}\right)$.

So we can get the Lebesgue constant of univariate rational function

$$
\Lambda_{n}=\max _{a \leq x \leq b} \sum_{k=0}^{n}\left|l_{k}(x)\right|=\max _{a \leq x \leq b} \frac{\sum_{k=0}^{n}\left|\frac{w_{k}}{x-x_{k}}\right|}{\left|\sum_{k=0}^{n} \frac{w_{k}}{x-x_{k}}\right|} .
$$

The Lebesgue constant of the univariate rational function be generalized to the bivariate case as follows

$$
\Lambda_{m, n}=\max _{a \leq x \leq b, c \leq y \leq d} \frac{\sum_{i=0}^{m} \sum_{j=0}^{n}\left|\frac{w_{i} \cdot u_{j}}{\left(x-x_{i}\right)\left(y-y_{j}\right)}\right|}{\left|\sum_{i=0}^{m} \sum_{j=0}^{n} \frac{w_{i} \cdot u_{j}}{\left(x-x_{i}\right)\left(y-y_{j}\right)}\right|} .
$$


Bivariate barycentric rational interpolant is determined when weights are given. The key question is how to choose weights by minimizing the Lebesgue constant. The weighs are been as decision variables and satisfy some constraint conditions to ensure the bivariate barycentric rational function has no poles and no unattained points and uniqueness.

The optimization model for optimal interpolation weights be constructed as follows

$$
\begin{aligned}
& \min =\max _{a \leq x \leq b, c \leq y \leq d} \overline{\sum_{i=0}^{m} \sum_{j=0}^{n}\left|\frac{w_{i} \cdot u_{j}}{\left(x-x_{i}\right)\left(y-y_{j}\right)}\right|}, \\
& \operatorname{sign}\left(w_{i}\right) \cdot \operatorname{sign}\left(w_{i+1}\right)=-1, i=0,1, \cdots, m, \\
& \operatorname{sign}\left(u_{j}\right) \cdot \operatorname{sign}\left(u_{j+1}\right)=-1, j=0,1, \cdots, n, \\
& w_{i} \neq 0, i=0,1, \cdots, m, \\
& u_{j} \neq 0, j=0,1, \cdots, n, \\
& \sum_{i=0}^{m}\left|w_{i}\right|=1 \\
& \sum_{j=0}^{n}\left|u_{j}\right|=1 .
\end{aligned}
$$

The optimal weights can be obtained by LINGO software.

\section{Numerical examples}

Example 4.1. Let $f_{1}(x, y)=\frac{1}{1+x^{2}}+\frac{y}{1+y^{2}}$ as the interpolated function and $x_{0}=0, x_{1}=0.25, x_{2}=0.5$, $y_{0}=0.5, y_{1}=0.75, y_{2}=1$ as interpolation points.

We can get the optimal barycentric weights by the LINGO software as follows

$$
\begin{aligned}
& w_{0}=0.2484435, w_{1}=-0.4968681, w_{2}=0.2546883 \\
& u_{0}=-0.2500033, u_{1}=0.4999975, u_{2}=-0.2499992
\end{aligned}
$$

In order to show the effectiveness of the new method, we give figures of the interpolation errors (see Fig. 4, Fig. 5), bivariate barycentric rational interpolant (see Fig. 2) and the interpolation function with the method in paper [10] (see Fig. 3) by software MATLAB.

Example 4.2. Let $f_{2}(x, y)=\sin \left(x+y^{2}\right)$ as the interpolated function and let $x_{0}=0, x_{1}=0.25, x_{2}=0.5$ and $y_{0}=0.5, y_{1}=0.75, y_{2}=1$ as the interpolation points.

We can also get optimal barycentric weights by the LINGO software as follows

$$
\begin{aligned}
& w_{0}=0.2484435, w_{1}=-0.4968681, w_{2}=0.2546883 \\
& u_{0}=-0.2500033, u_{1}=0.4999975, u_{2}=-0.2499992
\end{aligned}
$$

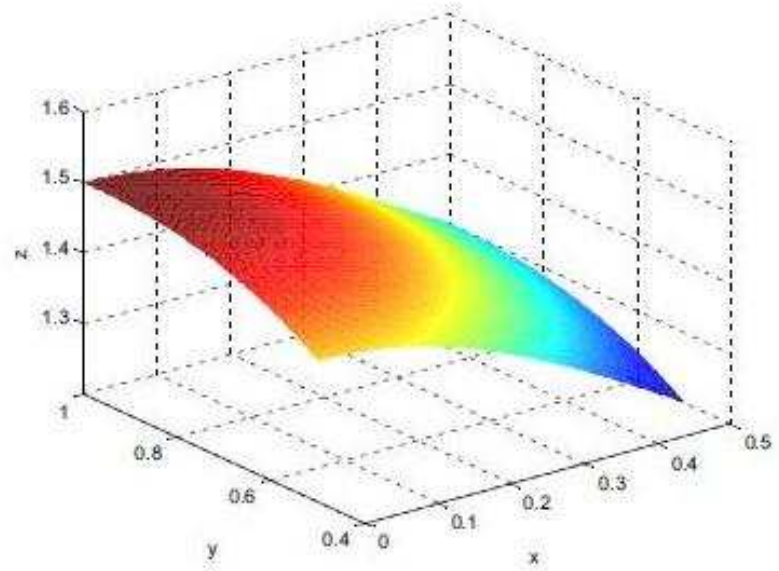

Fig. 1: The interpolated function $f_{1}(x, y)$.

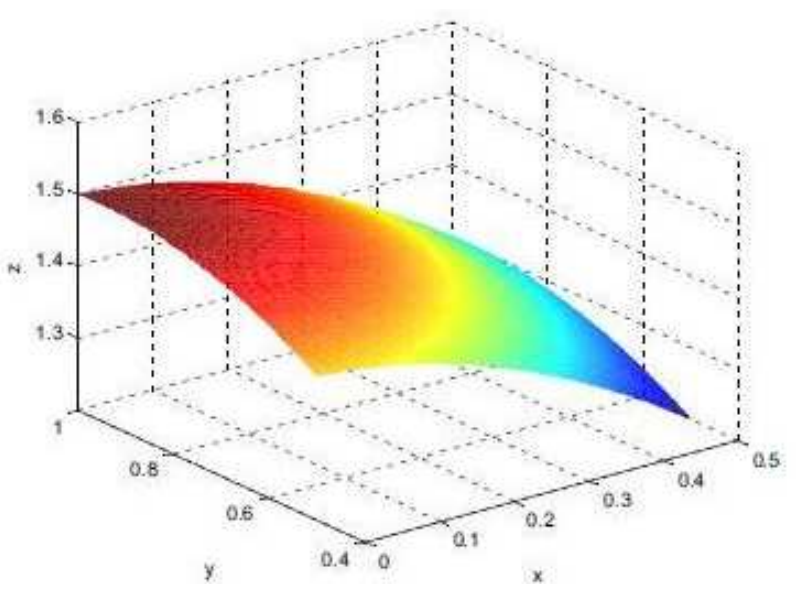

Fig. 2: The interpolation function with the new method.

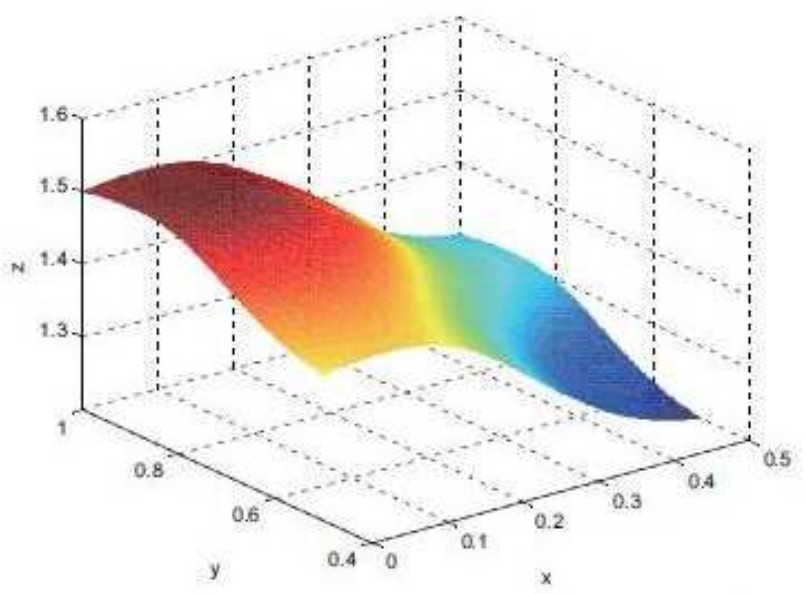

Fig. 3: The interpolation function with the method in paper [10]. 


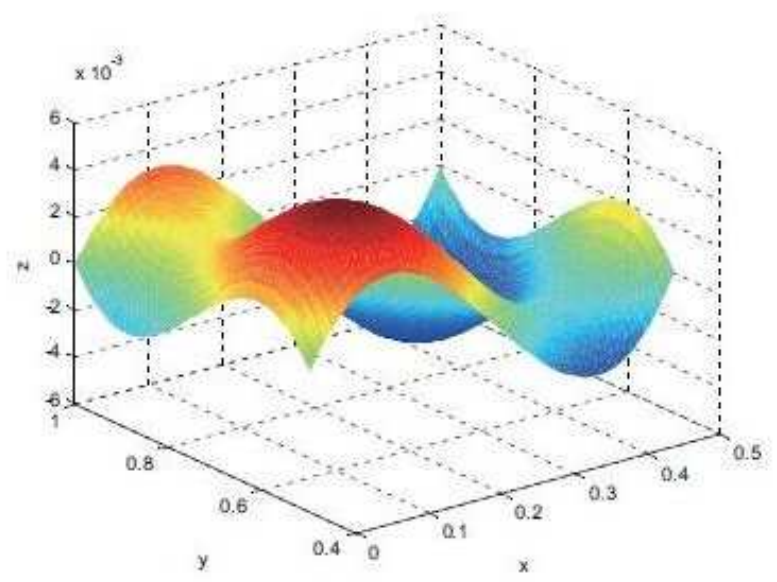

Fig. 4: The interpolation error with the new method.

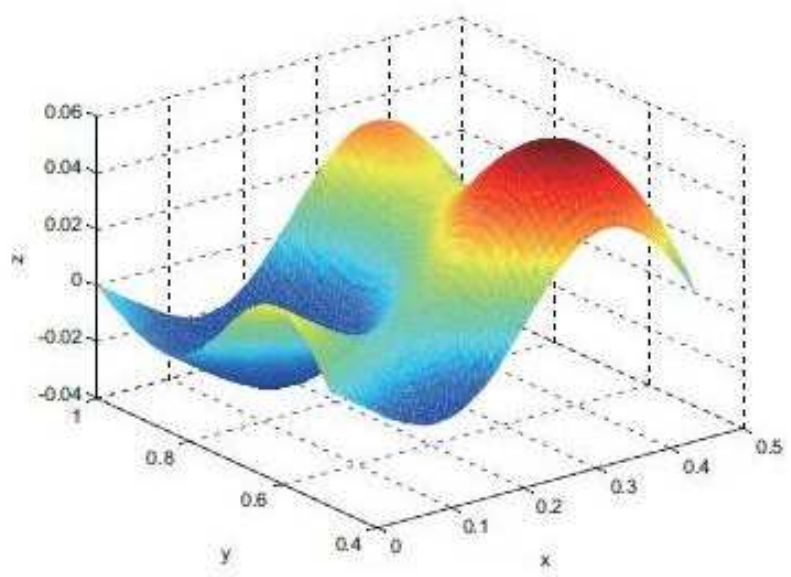

Fig. 5: The interpolation error with the method in paper [10].

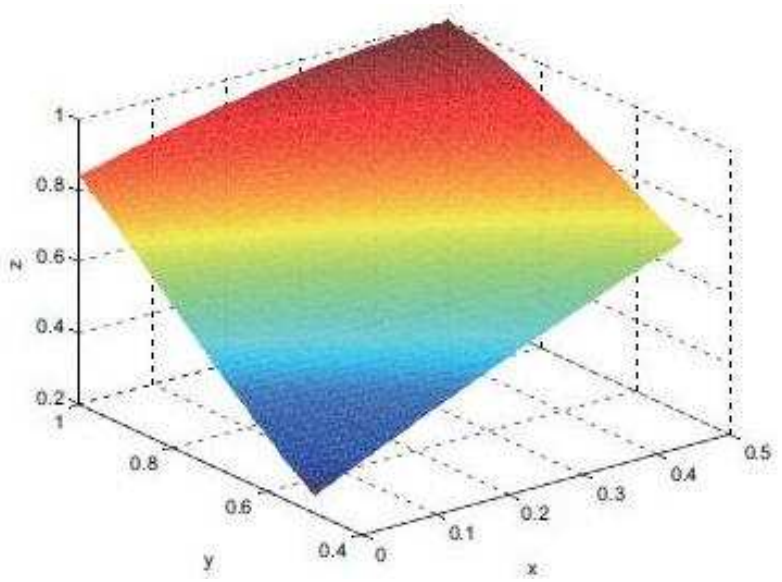

Fig. 6: The interpolated function $f_{2}(x, y)$.

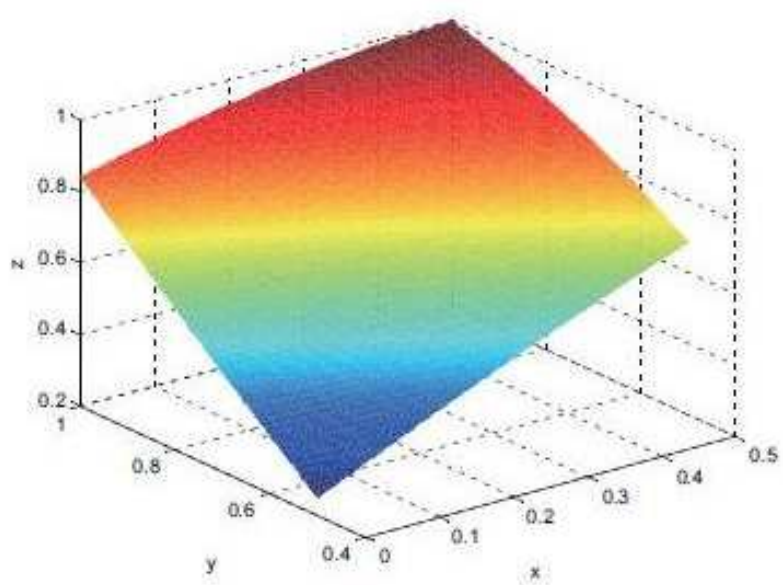

Fig. 7: The interpolation function with the new method.

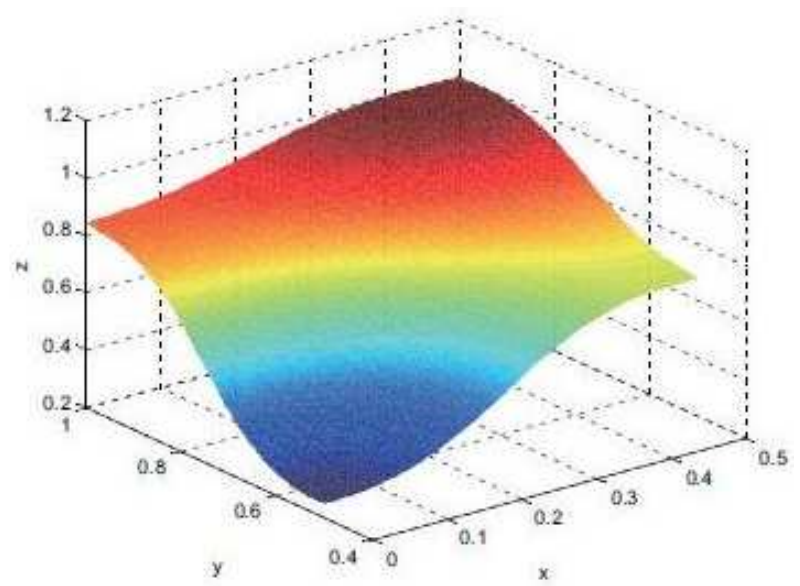

Fig. 8: The interpolation function with the method in paper [10].

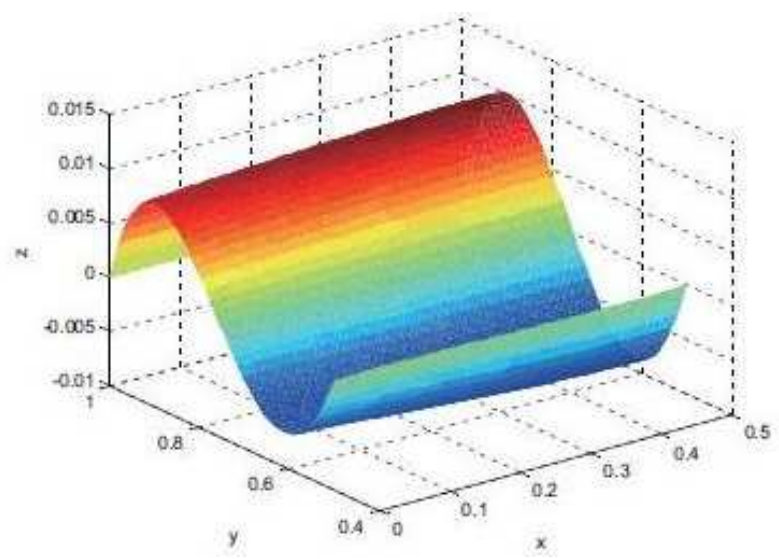

Fig. 9: The interpolation error with the new method. 


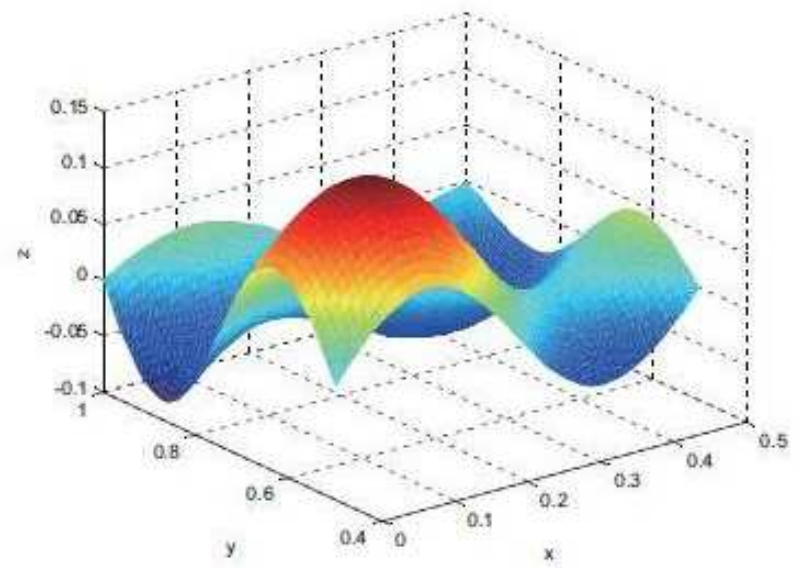

Fig. 10: The interpolation error with the method in paper [10].

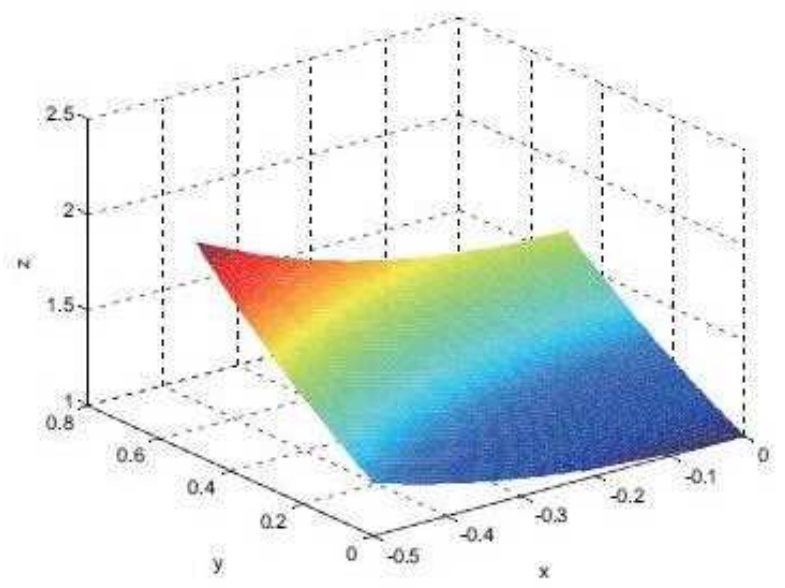

Fig. 11: The interpolated function $f_{3}(x, y)$.

So some figures like above to show the effectiveness of the new method are shown in Figure 6, Figure 7, Figure 8, Figure 9 and Figure 10.

Example 4.3. Let $f_{3}(x, y)=e^{x^{2}+y}$ as the interpolated function. Let $x_{0}=-0.5, x_{1}=-0.25, x_{2}=0$ and $y_{0}=0, y_{1}=-0.25, y_{2}=0.5$ as the interpolation points.

We can get a set of optimal weights by software LINGO from the above optimization model.

$$
\begin{aligned}
& w_{0}=-2.772280, w_{1}=0.4818480, w_{2}=-0.2409240 \\
& u_{0}=0.2500000, u_{1}=-0.5000000, u_{2}=0.2500000
\end{aligned}
$$

We can also get some figures (see Fig. 11, Fig. 12, Fig. 13, Fig. 14 and Fig. 15) like above to show the effectiveness of the new method.

Obviously, the new method is better.

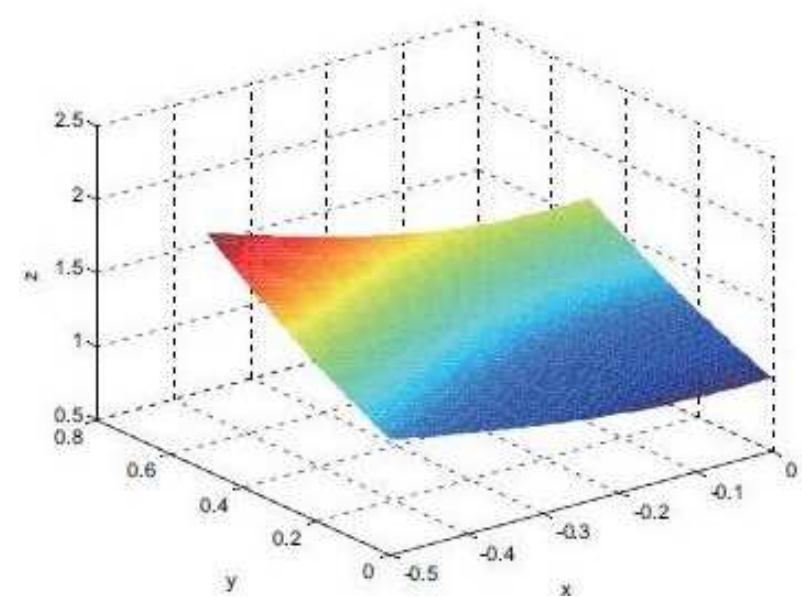

Fig. 12: The interpolation function with the new method.

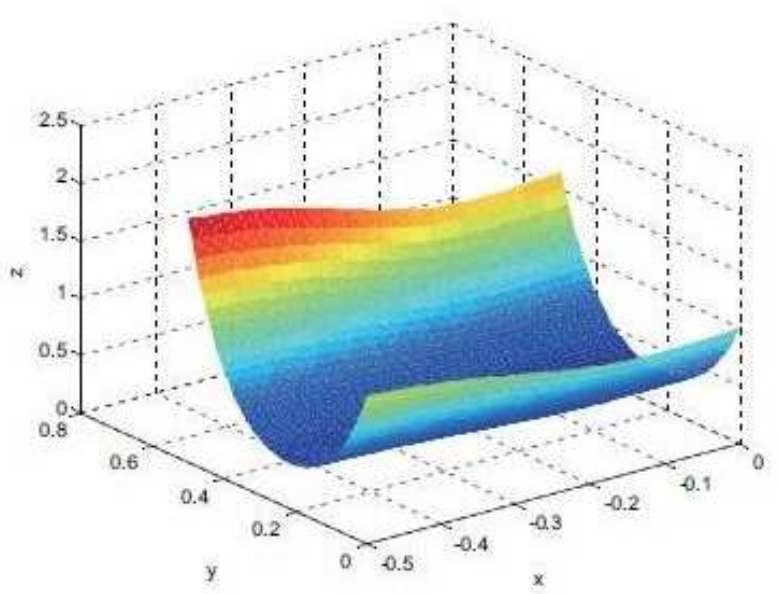

Fig. 13: The interpolation function with the method in paper [10].

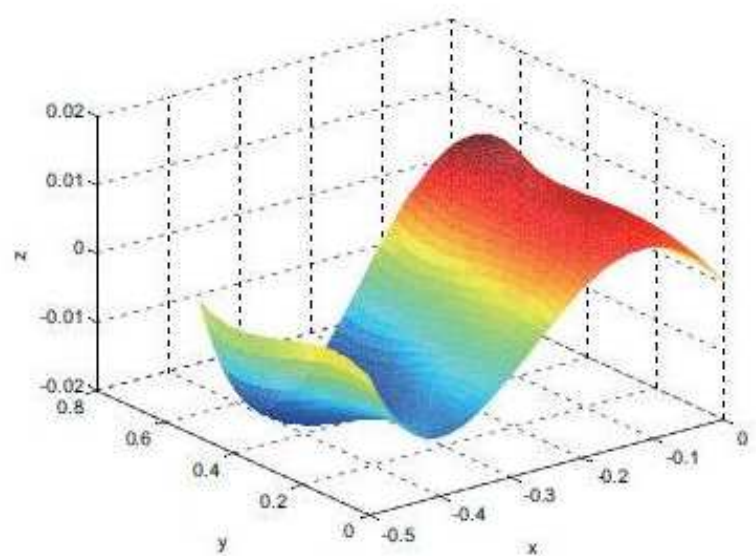

Fig. 14: The interpolation error with the new method. 


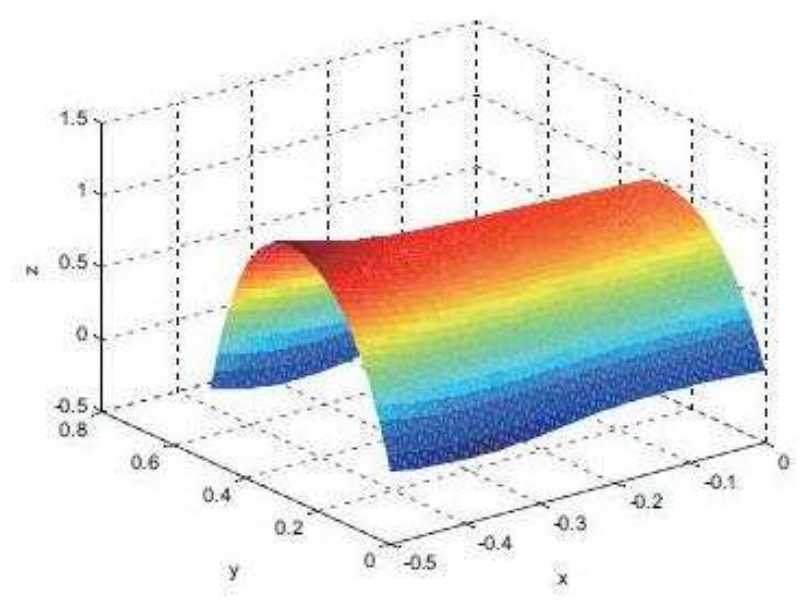

Fig. 15: The interpolation error with the method in paper [10].

\section{Conclusions}

In this paper, the optimization algorithm model for bivariate barycentric rational interpolation is studied. A new optimization algorithm is given for the best interpolation weights based on the Lebesgue constant minimizing. Minimizing Lebesgue constant is the objective function; the weights are decision variables and satisfy some constraint conditions to ensure the bivariate barycentric rational function has no poles and no unattained points and uniqueness. The solution of the optimization model is obtained using the software LINGO.

In future work, the shape control in bivariate barycentric rational interpolation based on this new method will be studied.

\section{Acknowledgements}

The authors wish to thank the helpful comments and suggestions from my teachers and colleagues in Service computing Lab. We would like to thank the support of the National Natural Science Foundation of China under Grant No.60973050, No.61272153, No.61170059 and No.61170172, the National High-Tech Research and Development Plan of China under Grant No.2009AA01Z401, the Natural Science Foundation of Educational Government of Anhui Province of China (KJ2009A50, KJ2007B173, KJ2012A073, KJ2011A086), Anhui Provincial Natural Science Foundation (1208085MF105), Anhui Provincial Soft Science Foundation(12020503031), Program for Excellent Talents in Anhui and Program for New Century Excellent Talents in University(NCET-06-0555).

\section{References}

[1] L. Knockaert, A simple accurate algorithm for barycentric rational interpolation, IEEE Signal processing letters, 15, 156-157 (2008).

[2] H. T. Nguyen, A. Cuyt and O. S. Celis, Comonotone and coconvex rational interpolation and approximation, Numerical Algorithms, 58, 1-21 (2010).

[3] S. M. Floater and H. Kai, Barycentric rational interpolation with no poles and high rates of approximation, Numerische Mathematik, 107, 315-331 (2007).

[4] J. P. Berrut, R. Baltensperger and H. D. Mittclmann, Recent developments in barycentric rational interpolation, Interpolation Series of Nnmerical Mathematics, 151, 27-51 (2005).

[5] C. Schneider and W. Werner, Some new aspects of rational interpolation, Mathematics of Computation, 47, 285-299 (1986).

[6] J. P. Berrut and H. D. Mittelmann, Lebesgue constant minimizing linear rational interpolation of continuous function over the interval, Computers \& Mathematics with Applications, 33, 77-86 (1997).

[7] L. Bos, H. D. Marchi and M. Vianello, On the Lebesgue constant for the $\mathrm{Xu}$ interpolation formula, 141, 134-141 (2006).

[8] S. J. Smith, Lebesgue constants in polynomial interpolation, Annales Mathematics et Informaticae, 33, 109-123 (2006).

[9] H. T. Nguyen, A. Cuyt and O. S. Celis, Shape control in multivariate barycentric rational interpolation, Proc. ICNAAM 2010, 1281, 543-548 (2010).

[10] J. P. Berrut, Rational functions for guaranteed and experimentally well-conditioned global interpolation, Computers \& Mathematics with Applications, 15, 1-16 (1988).

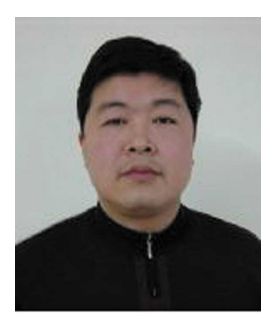

Qianjin Zhao received the Ph.D. degree from Hefei University of Technology, China. $\mathrm{He}$ is currently a professor in School of Science at Anhui University of Science and Technology. His research interests are in the areas of rational interpolation, digital image processing and computer-aided geometric design.
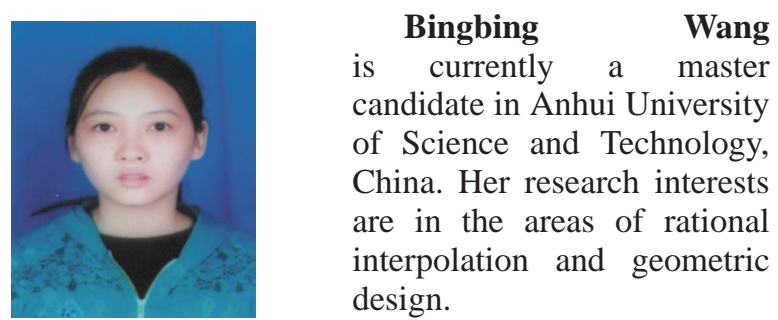